T cells. It is also expressed on Treg cells, thereby contributing to the development of peripheral tolerance. Semaphorins, a family of axon guidance molecules during nervous system development, have recently found to be involved in regulating the immune system. To explore the possible involvement of NP-1 and Semaphorin's in the development of lupus nephritis (LN).

Methods Twelve kidney biopsies from LN patients (8 - diffuse proliferative and 4 - focal) and five normal biopsies were enrolled in this study. They were subjected to immunohistochemistry staining with anti VEGF, NP-1, Semaphorin 3A, $4 \mathrm{~A}$ and 4D. The histological slides were scanned by Olympus BX51 microscope and digitised using a digital camera. The Image Pro-Plus 6.3 program was used to measure the intensity and extent of staining. The correlation between the staining and the clinical variables (serum creatinine, proteinuria, activity and chronicity index) was evaluated.

Results NP-1 and Semaphorin's, were stained with significantly higher intensity in the LN group (diffuse more than focal) than the control group. VEGF staining in the glomeruli slightly increased compared to the control group. In focal LN, NP-1 deposition was found to be limited to diseased glomerular area, whereas in diffuse proliferative LN, NP-1 was diffusely expressed. NP-1 was found to be correlation with clinical parameters (statistical trend), whereas Semaphorin's were found in inverse statistically significant correlations.

Discussion This is the first study to reporting on the involvement of NP-1 and immune Semaphorin's in LN. NP-1 is suggested to become a histological marker for focal versus diffuse LN. In addition it is a reliable marker for LN severity. Being present in normal glomeruli, but slightly increased in LN, VEGF could be considered as a compensatory protective factor during inflammation. As a functional VEGF receptor, one can assume that increased NP-1 expression in LN is meant to intensify the possible protective effect of VEGF on the glomerular endothelial cells, aiming to prevent their damage and apoptosis. Semaphorin 3A a known destabiliser of glomerular barrier and a VEGF competitor (a NP-1 ligand), reducing thereby its protective effects and enhancing proteinuria. The inverse correlation could be the result of the altered ability of kidneys to secrete Semaphorin's during advanced renal damage. Further work is needed in order to explore the entire scenario of the involvement of the NP-1 and Semaphorin's in LN.

\title{
A110 THE INVOLVEMENT OF NEUROPILIN-1 AND IMMUNE SEMAPHORIN'S IN LUPUS NEPHRITIS
}

Vadasz Zahava, ${ }^{1}$ Ben-Izhak Ofer, ${ }^{2}$ Bejar Jacob, ${ }^{3}$ Sabo Edmond, ${ }^{2}$ Storch Shimon, ${ }^{4}$ Toubi Elias ${ }^{1}$ Division of Allergy and Clinical Immunology, Bnai-Zion Medical Center, Haifa, Israel; '2Department of Pathology, Rambam Medical Center, Haifa, Israel; ${ }^{3}$ Department of Pathology, Bnai-Zion Medical Center, Rappaport faculty of Medicine, the Technion, Haifa, Israel; ${ }^{4}$ Nephrology Unit, Bnai-Zion Medical Center, Rappaport faculty of Medicine, the Technion, Haifa, Israel

10.1136/ard.2010.148981.13

Background and objectives Neuropilin-1(NP-1), a known vascular endothelial growth factor (VEGF) receptor, is important to the interaction between activated DCs and resting 